\title{
Pulmonary Artery Pseudoaneurysms in the Setting of Gout Polyarthropathy: A Case Report
}

\author{
Amelia Bai ${ }^{\mathrm{a}}$, Ling Lan ${ }^{\mathrm{b}, \mathrm{e}}$, Karl Poon ${ }^{\mathrm{c}}$, Varghese Pynadath Joseph ${ }^{\mathrm{d}}$, \\ Rachael O'Rourke ${ }^{d}$, Khurrum Aftab ${ }^{d}$
}

\begin{abstract}
Pulmonary artery pseudoaneurysm is a dangerous pathology often missed as a differential and on imaging. With a 50\% mortality rate early detection and intervention are critical to patient outcome, rendering diagnosis based on clinical grounds to be of paramount importance. A high index of suspicion should be placed on patients presenting with unprovoked hemoptysis, hemorrhage or hemothorax. Knowledge of the common causes of pulmonary artery pseudoaneurysms is also important; however, it is integral to note spontaneous occurrences in patients with no known risk factors. Herein, we report a case of an unprovoked pulmonary artery pseudoaneurysm in a 72-year-old male with no known risk factors or causes for the pathology. Additionally, we review the pathophysiology behind the potential association of polyarthritic gout as a cause of pulmonary artery pseudoaneurysms. To the best of our knowledge this is the first reported link between the two diseases, providing grounds to widen literature and increase diagnoses of pulmonary artery pseudoaneurysms.
\end{abstract}

Keywords: Pulmonary artery pseudoaneurysms; Embolization; Gout polyarthropathy

\section{Introduction}

Pulmonary artery pseudoaneurysm is a rare and potentially life threatening pathology. Not to be confused with pulmonary

Manuscript submitted June 12, 2018, accepted July 25, 2018

aUniversity of Queensland Exchange Program, Medical School of Flinders University, South Australia, Australia

${ }^{\mathrm{b}}$ Geriatric and Rehabilitation of Internal Medicine Services, The Prince Charles Hospital, Chermside, QLD 4032, Australia

${ }^{\mathrm{c}}$ Cardiology Department, The Prince Charles Hospital, Chermside, Affiliated With School of Medicine, University of Queensland, QLD 4032, Australia dRadiology Department, The Prince Charles Hospital, Chermside, QLD 4032, Australia

${ }^{\mathrm{e}}$ Corresponding Author: Ling Lan, Geriatric and Rehabilitation of Internal Medicine Services, The Prince Charles Hospital, Affiliated With School of Medicine, University of Queensland, Chermside, QLD 4032, Australia. Email: ling.lan@health.qld.gov.au

doi: https://doi.org/10.14740/jocmr3513w artery aneurysms, pseudoaneurysms do not involve all layers of the arterial wall, putting them at higher risk of rupture [1]. They most commonly arise via iatrogenic causes such as Swan-Ganz or Cournand catheter complications, chest tube insertions, and pulmonary biopsies [2]. Other acquired causes include infections such as bacterial endocarditis and tuberculosis (Rasmussen aneurysm), carcinoma, congenital heart disease, necrotizing pneumonia and vasculitis (as in Behcet's disease). Reported risk factors include pulmonary hypertension, chronic steroid use, anticoagulation therapy, cardiac manipulation during surgery, age $>60$ years, and female sex [3].

Hemoptysis is the most common presenting complaint and a pseudoaneurysm may be an incidental finding on chest X-ray (CXR), computed tomography (CT) or magnetic resonance imaging (MRI). Alternatively, patients may present with acute severe hemorrhage or hemothorax [4]. Definitive diagnosis is made by pulmonary artery angiography, however CXR may show a mass which can be further evaluated with high-resolution CT or MRI angiography [2]. If left untreated pseudoaneurysms can rupture and cause exsanguination, leading to death in approximately $50 \%$ of patients [3]. This high mortality rate calls for prompt recognition and therapy.

Endovascular treatment by direct coil embolization (stainless steel or platinum), stent placement, or embolization of the feeding vessel remains the treatment of choice [5]. All are effective in occluding pseudoaneurysms and provide an effective and safe alternative to surgical treatment options such as wedge resection or lobectomy [2].

We present the case of a patient with pulmonary artery pseudoaneurysm, who as from the literature available had no apparent cause for the abnormality. We investigate whether a community acquired pneumonia or gout causing chronic inflammation could be the cause for his spontaneous pseudoaneurysm.

\section{Case Report}

A 72-year-old man presented to hospital with right-sided chest pain. He was admitted for evaluation of non-ST elevation MI (troponin 0.097), fever, and right lower zone community acquired pneumonia with parapneumonic effusion. Past medical history revealed non-ischemic cardiomyopathy (NYHA2) with an ejection fraction of 33\%. Apart from an angiogram which was done 4 months prior to this presentation, no other invasive procedures had been conducted recently. The patient 


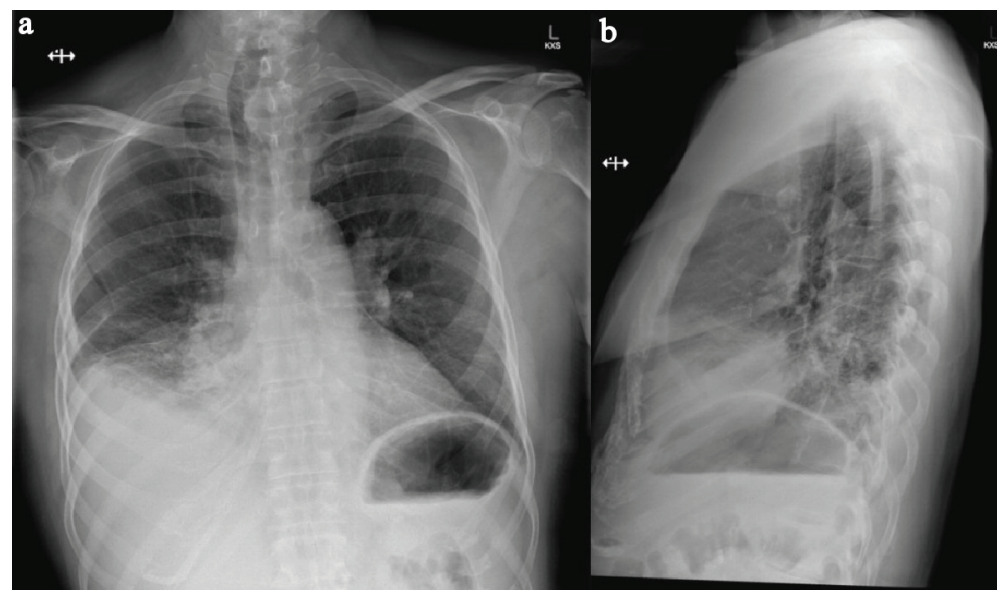

Figure 1. (a) Erect PA chest X-ray showing consolidation and atelectatic changes of right middle and lower lobes obscuring hemidiaphragm with associated mild effusion. (b) Lateral view of same chest X-ray. Note: trachea displaced to the right consistent with known left retrosternal thyroid goitre.

also had a left-sided stroke 10 years ago. Other co-morbidities included hypertension, hyperlipidemia, type 2 diabetes mellitus, peripheral vascular disease, gout, bilateral internal carotid artery stenosis, moderate alcohol consumption and previous smoking history. During admission he had recurrent gout polyarthropathy flare ups requiring colchicine $(0.5 \mathrm{mg})$ daily. There was no history of bacterial endocarditis, exposure to tuberculosis, congenital heart disease or vasculitis.

Significant laboratory results included elevated white blood cells $\left(19.6 \times 10^{9} / \mathrm{L}\right)$, neutrophils $\left(14.67 \times 10^{9} / \mathrm{L}\right)$ and gamma-glutamyl transferase (96 U/L). CXR confirmed consolidation of right middle and lower lobes with loss of outline of right hemi-diaphragm suggesting mild to moderate right pleural effusion (Fig. 1). Ongoing hypoxia and increased dyspnoea, associated with hypoactive delirium and elevated CRP called for ultrasound guided pleural drainage and intercostal

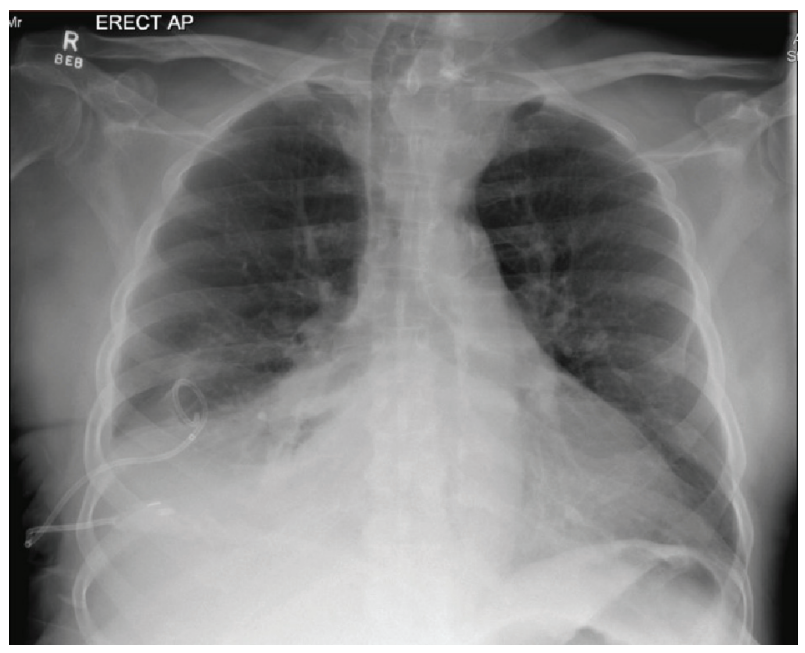

Figure 2. Erect AP chest X-ray showing newly inserted right-sided chest drain in situ, collapse of right lung base and moderate resolution of right-sided pleural effusion. Remaining lungs are clear. Vessels in both hila noted to be prominent. Upper lobe redistribution of pulmonary flow can be seen. catheter. Thoracocentesis produced freely draining hemorrhagic effusion, and on the same day CXR showed moderate resolution of the right hemothorax (Fig. 2). Pleural fluid was collected with no malignant cells seen, and had no bacterial or fungal growth on culture. The patient was commenced on IV antibiotics. Two days after the intercostal catheter was inserted drain output increased, and the patient remained febrile despite continued negative blood cultures. A CT chest angiogram was requested to exclude any rib fractures, pulmonary embolism or active bleeding. Findings reported from CT chest included large right-sided pleural collection with adjacent atelectasis (Fig. 3), no contrast extravasation through intercostal vessels to suggest active bleeding, no pulmonary embolism, no rib fracture, and no filling defect of main pulmonary arteries. A pseudoaneurysm was missed in this initial report with specific comment on no filling defect in right lobar and segmental branches of the main pulmonary artery.

Two days after initial CT the right pleural effusion recollected, supported by CXR, a further $1 \mathrm{~L}$ of aspirate was drained from the intercostal catheter. Four days after the initial CT the patient remained febrile despite continued IV antibiotics and absence of ongoing drainage from the intercostal catheter. Ultrasound examination revealed small volume right pleural

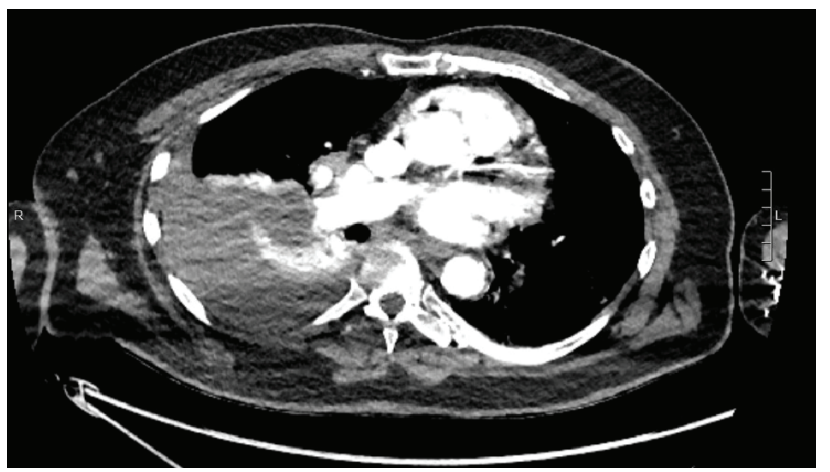

Figure 3. Thin slice from CT pulmonary angiogram showing large rightsided pleural collection (clinically hemothorax). 


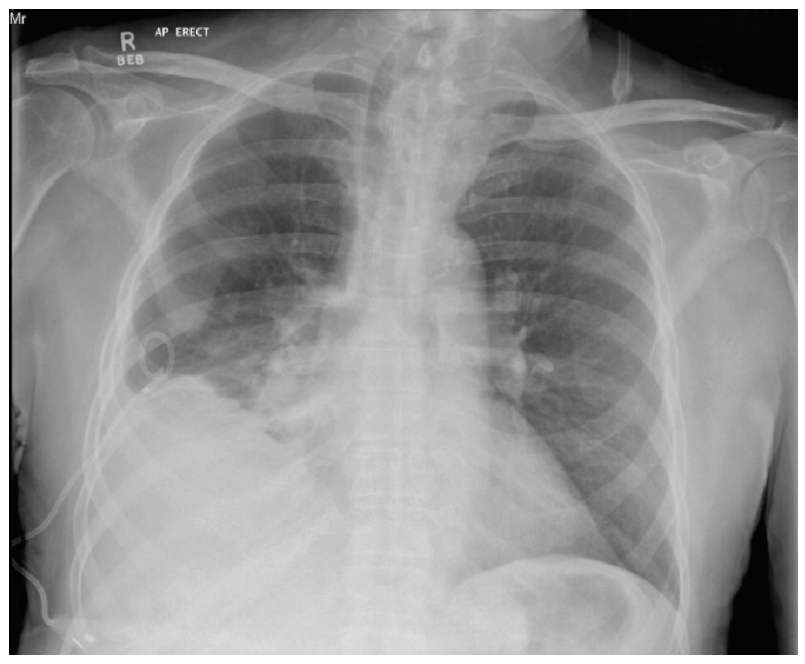

Figure 4. AP erect chest $\mathrm{X}$-ray 7 days after intercostal catheter insertion for right pleural effusion. No ongoing drainage. Evidence of clearing of parenchymal changes compared to initial X-rays (Fig. 1).

effusion of approximately $200 \mathrm{~mL}$ and repeat CXR showed residual costophrenic angle effusion and clearing of parenchymal changes in right lower lobe (Fig. 4). The intercostal catheter was removed and it was decided to perform a second CT chest to exclude ongoing effusion or pneumothorax. Repeat CT chest on day 12 of the patient's admission revealed a large, rounded contrast abnormality contiguous with the right medial segmental branch of the middle lobe pulmonary artery, most likely representing a pseudoaneurysm (Fig. 5). This was a new finding as compared to CTPA examinations from 12 months prior. When compared to the previous CT 4 days prior the mass had increased in size by $5 \mathrm{~mm}$ on the coronal plane. In order to define the contrast abnormality seen on CT, a MRI chest was ordered (Fig. 6). A large round pseudoaneurysm arising from the right medial segmental branch of the middle lobe pulmonary artery was confirmed, and steady state free precession sequences imaging demonstrated flow between the right pulmonary artery and the lesion.

On day 19 of admission, the patient underwent pulmonary artery pseudoaneurysm embolization. An angiogram was performed before and after the procedure, with final results revealing complete occlusion of the aneurysm (Fig. 7). Access into the right pulmonary artery was gained using a pigtail catheter and J-wire, and a right pulmonary angiogram was performed. The pseudoaneurysm and its neck were best demonstrated in the LAO 55 degree projection. The anatomy of the pseudoaneurysm was delineated. Access was obtained into the pseudoaneurysm using a $\mathrm{C} 2$ catheter and Progreat microcatheter. Embolization was then performed with multiple detachable Concerto coils (Fig. 7). The procedure was well tolerated by the patient and no immediate complications arose. Follow-up of the patient months later revealed no pulmonary complications and the patient remains symptom free. His only concerns now are the sequelae of his pre-existing non-ischemic cardiomyopathy.

\section{Discussion}

Pulmonary artery pseudoaneurysm is a rare pathology most commonly arising from iatrogenic sources. Other well documented causes include tuberculosis and Behcet's syndrome [2]. In the literature hemoptysis is the most common presenting complaint, and signs of rupture such as hemothorax

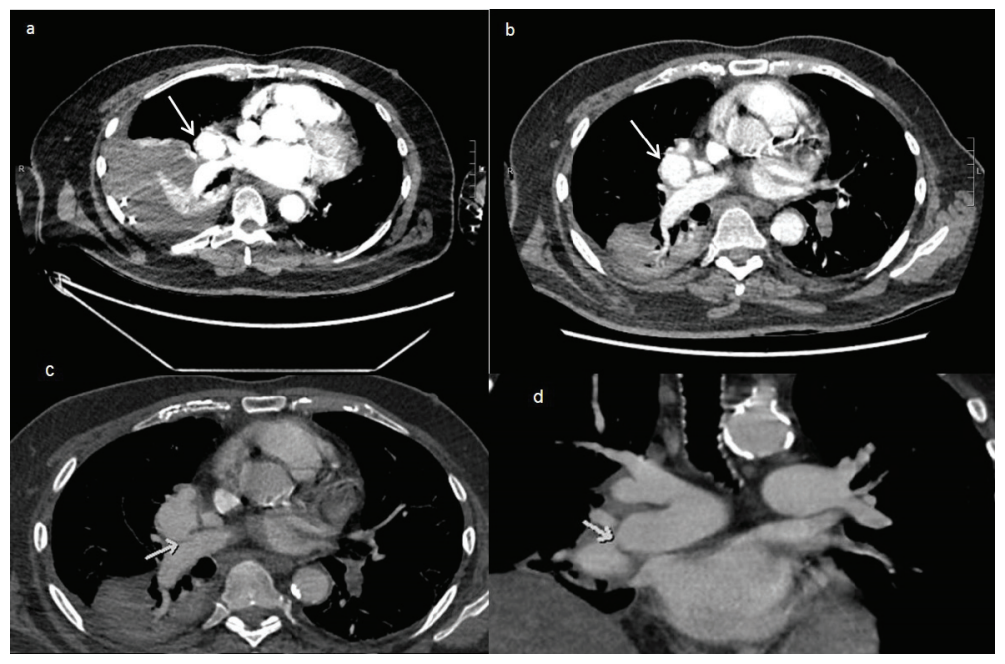

Figure 5. (a) CT pulmonary angiogram on day 6 of patient presentation showing a pulmonary artery pseudoaneurysm (arrow) measuring $35 \times 32 \times 35 \mathrm{~mm}($ AP $\times$ Transverse $\times \mathrm{CC}$ ). Note presence of large right-sided pleural collection with adjacent atelectasis. (b) CT pulmonary angiogram on day 12 of patient presentation showing growth of the pseudoaneurysm (arrow), measuring $39 \times 35 \times 40 \mathrm{~mm}$. There is a decrease in the size of the right pleural collection, showing a slightly loculated appearance. (c) Axial post contrast CT showed a large pseudoaneurysm arising from the distal right main pulmonary artery with homogenous contrast filling. The arrow showed the small connection between the pseudoaneurysm and the right pulmonary artery. (d) Coronal post contrast CT demonstrating the large pseudoaneurysm arising from the distal right main pulmonary artery (arrow), abutting the left atrium and right hemidiaphragm. 


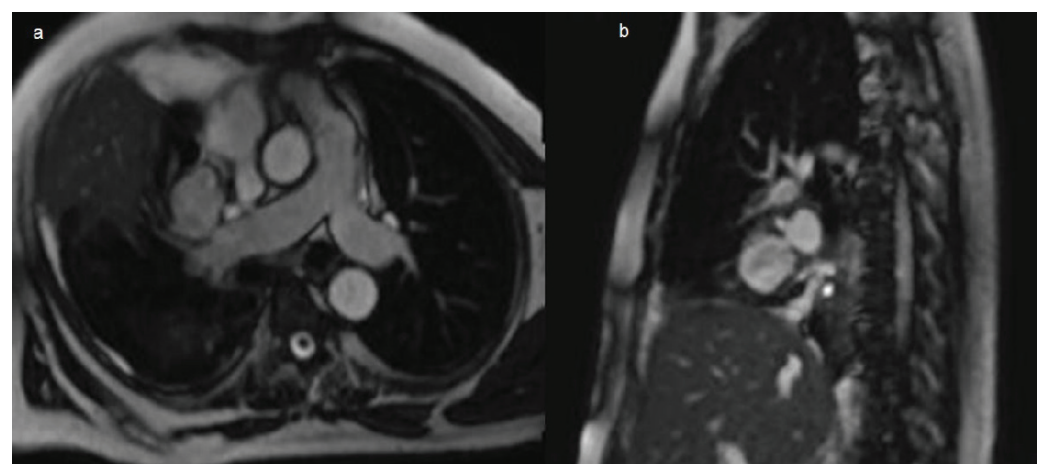

Figure 6. (a) Chest axial oblique non contrast MRI steady state free precession sequences showing a right pulmonary artery pseudoaneurysm arising from the medial segmental branch of the middle lobe, measuring $37 \times 32 \times 37 \mathrm{~mm}$. (b) Sagittal imaging through the pseudoaneurysm confirming connection to the right pulmonary artery.

are associated with $50 \%$ mortality [3]. We report the case of a 72-year-old man with multiple comorbidities who was diagnosed with a pulmonary artery pseudoaneurysm despite having no hemoptysis and no clear underlying cause for the abnormality. Our patient fulfilled none of the documented etiology of pseudoaneurysms including tuberculosis, invasive chest procedures, neoplasm, vasculitis, infective endocarditis, or pulmonary arterial hypertension [2]. This begs the investigation for a possible cause for our patient's newly acquired pulmonary artery pseudoaneurysm.

Important to acknowledge is our patient's multiple cardiovascular risk factors. Despite this he did not have known pulmonary artery hypertension as sequelae to non-ischemic cardiomyopathy. Additionally, literature states that pulmonary artery hypertension more commonly causes pulmonary artery aneurysms than pseudoaneurysms [2]. Therefore it is unlikely our patient's cardiovascular status contributed to the formation of the new pulmonary artery pseudoaneurysm.

Previously reported in literature is an association between necrotizing pneumonia and pulmonary artery pseudoaneurysms [3-5]. In our patient it is difficult to determine whether the community acquired pneumonia was the cause of the pseudoaneurysm or whether it was superimposed with the presentation. One aspect we can confirm is that the presumed infection-caused pleural effusion was not due to the pneumonia, but rather due to the pseudoaneurysm. Not only did it not resolve with IV antibiotics, but culture of the fluid remained negative for any organisms. Additionally, in the reported cases of pulmonary artery pseudoaneurysms caused by bacterial pneumonia, all patients who received IV antibiotics showed evidence of resolution or decrease in size of the pseudoaneurysm on follow-up CT studies [5]. Our patient, however, showed growth on follow-up CT scans despite continued IV antibiotics and resolution of pulmonary consolidation. This is in keeping with our suggestion that the pneumonia was not the primary cause of the pulmonary artery pseudoaneurysm, but rather a superimposed pathology.

A cause we would like to suggest is the association between chronic inflammation (from gout) and pulmonary artery pseudoaneurysms. Well documented is the pathogenesis of Behcet's disease and its associated pulmonary manifestations. In Behcet's disease it is common to observe infiltrative

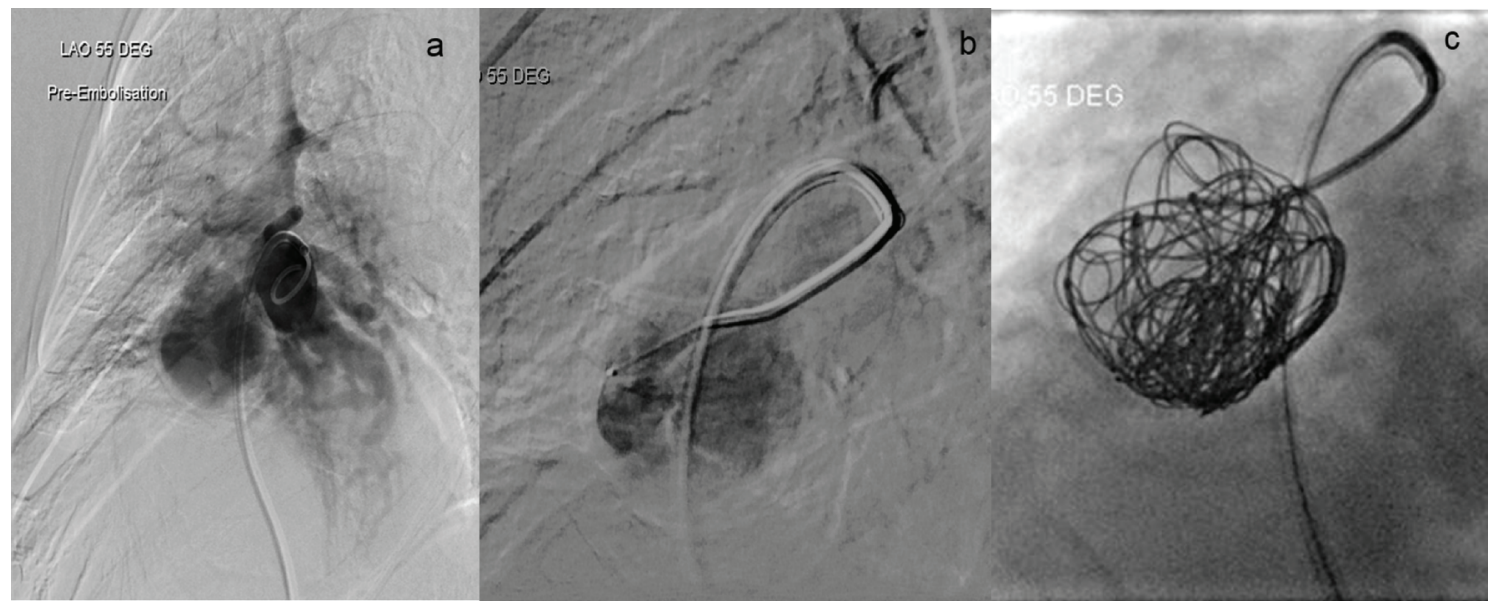

Figure 7. Digital subtraction angiography. (a) Pre-embolization angiogram demonstrating contiguous flow between the pseudoaneurysm and the middle lobe artery. (b) The microcatheter and contrast within the lumen of the pseudoaneurysm. (c) Final post coiling imaging revealing complete occlusion of the pseudoaneurysm after successful embolization with Concerto coils without discernible residual contrast filling. 
granulation tissue and necrotic intra-alveolar hemorrhages throughout the lung. The consequential intimal thickening and degeneration in elastic lamina of pulmonary arteries is thought to provide an area of weakness for an aneurysm or pseudoaneurysm to arise [6]. Although not a vasculitis, gout is classified as a chronic inflammatory condition. Its pathogenesis has previously been investigated and has been shown to promote interleukin production [7]. The same interleukin shown to be activated through the production of monosodium urate crystals, interleukin-1 beta (IL-1 $\beta$ ), has also been implicated in Behcet's disease; representing a clear role in triggering autoinflammation [8-10]. Additionally, it has been reported that high levels of serum uric acid (as in gout) also activate the NALP3 inflammasome, leading to IL-1 $\beta$ provoked lung inflammation [11]. It is through this association that we propose the chronic inflammatory state our patient was in, as a consequence of severe gout, may have played a role in the development of his pulmonary artery pseudoaneurysm. This is, to the best of our knowledge, the first reported potential association between gout and pulmonary artery pseudoaneurysms.

Lastly, we note that the diagnosis in our patient was missed on the initial CT examination, only to be discovered on re-imaging due to ongoing fevers despite cessation of drainage from the intercostal catheter. Literature states that pulmonary artery pseudoaneurysms are commonly missed, many of which are detected in retrospect or follow-up studies [5]. With this in mind we hope to increase awareness of the clinical and radiographic features of pulmonary artery pseudoaneurysms, emphasising that an underlying cause may be unclear and that hemoptysis is not always the presenting complaint.

\section{Disclosures}

Verbal and written informed consent was obtained from the patient for his de-identified information to be published in this article.

\section{Funding Support}

No agency or funding from any source was received for this work.

\section{Conflict of Interest}

None.

\section{References}

1. Nguyen ET, Silva CI, Seely JM, Chong S, Lee KS, Muller NL. Pulmonary artery aneurysms and pseudoaneurysms in adults: findings at $\mathrm{CT}$ and radiography. AJR Am J Roentgenol. 2007;188(2):W126-134.

2. Lafita V, Borge MA, Demos TC. Pulmonary artery pseudoaneurysm: etiology, presentation, diagnosis, and treatment. Semin Intervent Radiol. 2007;24(1):119-123.

3. Pelage JP, El Hajjam M, Lagrange C, Chinet T, VieillardBaron A, Chagnon S, Lacombe P. Pulmonary artery interventions: an overview. Radiographics. 2005;25(6):16531667.

4. Sellke F, Del Nido P, Swanson S, Sabiston D, Spencer F. Sabiston \& Spencer surgery of the chest. 8th ed. Philadelphia, Pa.: Elsevier Saunders. 2010;145.

5. Chen Y, Gilman MD, Humphrey KL, Salazar GM, Sharma A, Muniappan A, Shepard JO, et al. Pulmonary artery pseudoaneurysms: clinical features and CT findings. AJR Am J Roentgenol. 2017;208(1):84-91.

6. Gul A, Yilmazbayhan D, Buyukbabani N, Lie JT, Tunaci M, Tunaci A, Inanc M, et al. Organizing pneumonia associated with pulmonary artery aneurysms in Behcet's disease. Rheumatology (Oxford). 1999;38(12):1285-1289.

7. Martinon F, Petrilli V, Mayor A, Tardivel A, Tschopp J. Gout-associated uric acid crystals activate the NALP3 inflammasome. Nature. 2006;440(7081):237-241.

8. Martinon F, Tschopp J. Inflammatory caspases: linking an intracellular innate immune system to autoinflammatory diseases. Cell. 2004;117(5):561-574.

9. Stojanov S, Kastner DL. Familial autoinflammatory diseases: genetics, pathogenesis and treatment. Curr Opin Rheumatol. 2005; 17(5):586-599.

10. Dinarello CA. Blocking IL-1 in systemic inflammation. J Exp Med. 2005;201(9):1355-1359.

11. Gasse P, Riteau N, Charron S, Girre S, Fick L, Petrilli V, Tschopp $\mathrm{J}$, et al. Uric acid is a danger signal activating NALP3 inflammasome in lung injury inflammation and fibrosis. Am J Respir Crit Care Med. 2009;179(10):903-913. 